\title{
Characteristic Equations for the Lasing Modes of Infinite Periodic Chain of Quantum Wires
}

\author{
Volodymyr O. Byelobrov ${ }^{1}$, Trevor M. Benson ${ }^{2}$, Ayhan Altintas ${ }^{3}$ and Alexander I. Nosich ${ }^{1}$ \\ ${ }^{1}$ Institute of Radiophysics and Electronics NASU, ul. Proskury 12, Kharkiv 61085, Ukraine \\ Tel: +380(57)7203782 e-mail:volodia.byelobrov@gmail.com \\ ${ }^{2}$ George Green Institute for Eelectromagnetics Research, University of Nottingham, NG7 2RD, UK \\ ${ }^{3}$ Bilkent University, Ankara 06800, Turkey
}

\begin{abstract}
In this paper, we study the lasing modes of a periodic open optical resonator. The resonator is an infinite chain of active circular cylindrical quantum wires standing in free space. Characteristic equations for the frequencies and associated linear thresholds of lasing are derived. These quantities are considered as eigenvalues of specific electromagnetic-field problem with "active" imaginary part of the cylinder material's refractive index - Lasing Eigenvalue Problem (LEP).

Keywords: eigenproblem, laser, microcavity, quantum wire, threshold, Floquet series.

\section{INTRODUCTION}

Today's technologies enable manufacturing of advanced light-emitting devices based on single or multiple quantum wires embedded in epitaxially grown semiconductor microcavities [1,2]. Advantages of quantum wires with respect to quantum wells include their better thermal stability, lower chemical reactivity, and higher mechanical strength. Besides, patterned and periodically etched layered structures offer new functionalities if used in the distributed Bragg reflectors due to their potential of the lateral light confinement.
\end{abstract}

\section{PROBLEM FORMULATION}

The considered resonator consists of the parallel to the $z$-axis and periodic along the $x$-axis circular cylinders see Fig. 1. The distance between cylinder centers is $p$ and their radii are $a$. Assuming that the electromagnetic field is time-harmonic $\left(\sim e^{-i \omega t}\right)$ and does not vary along the $z$-axis, we will study the LEP as a 2-D problem.

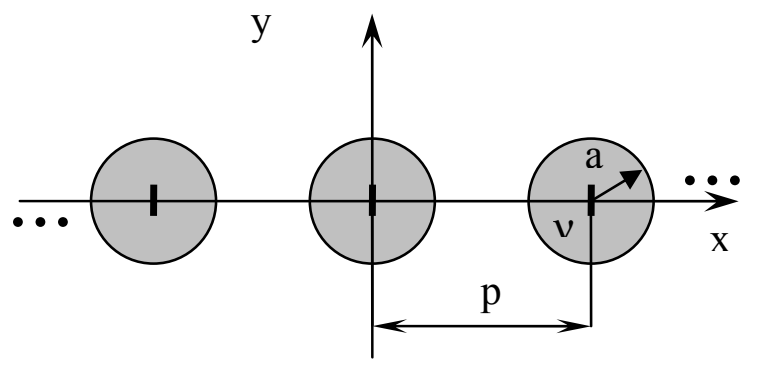

Fig. 1. Sketch of the periodic cavity of active dielectric circular cylinders or quantum wires.

Then two alternative polarizations, $\mathrm{E}$ and $\mathrm{H}$, can be considered separately using the function, $U(x, y)$, which will denote the $E_{z}$ or $Z_{0} H_{z}$ component of the electromagnetic field, respectively. It should satisfy the Helmholtz equation with different coefficients: $\left(k^{\prime}\right)^{2}=k^{2} v^{2}$, where $v=\alpha-i \gamma(\alpha, \gamma>0)$, inside each cylinder and $k^{2}$ outside. Here, $\alpha$ is the refractive index and $\gamma$ is the material gain which appears in the presence of pumping - see [3] for detailed discussion of the LEP approach in the linear modelling of microcavity lasers. Note also that it had been applied in the earlier papers [4,5] to the threshold analysis in the 2-D circular microcavities and in [6] to the similar analysis of the 1-D VCSEL-type layered cavity containing an active region.

The field components tangential to cylinder's boundary must be continuous. Besides, $U$ must satisfy the condition of the local power finiteness and the condition of radiation at infinity. Although the most general situation implies that the field functions within the adjacent elementary cells of the periodic cavity differ by a phase factor, i.e. $U(x+p, y)=U(x, y) e^{i \beta p}$, in the following treatment we will assume that $\beta=0$. The task is to find non-zero solutions to the formulated homogeneous problem. 


\section{BASIC EQUATIONS}

The periodicity condition means that internal fields of cylinders are equal to each other, and the contributions to the external field generated by each of cylinders are the same. Hence, on introducing the local polar coordinates co-axially with each cylinder as $\left(r_{s}, \varphi_{s}\right)$ and the global coordinates with the origin in the centre of the 0 -th cylinder as $(r, \varphi)$, we can use the partial separation of variables and write

$$
\begin{gathered}
U_{s}^{\mathrm{int}}\left(r_{s}, \varphi_{s}\right)=\sum_{(n)} c_{n}^{\prime} J_{n}\left(k^{\prime} r_{s}\right) e^{i n \varphi_{s}}, \quad r_{s}<a \\
U^{\text {out }}(r, \varphi)=\sum_{(s)} U_{s}^{\text {out }}\left(r_{s}, \varphi_{s}\right)=\sum_{(s)} \sum_{(n)} b_{n}^{\prime} H_{n}^{(1)}\left(k r_{s}\right) e^{i n \varphi_{s}}, \quad r_{s}>a
\end{gathered}
$$

where summations are performed from $-\infty$ to $+\infty$. Note that expressions (1) and (2) satisfy the Helmholtz equation and the conditions of radiation and local power finiteness.

The structure in Fig. 1 has two lines of symmetry, the $x$-axis and the $y$-axis. Therefore, one can separate the modes to independent symmetry families, to simplify the following analysis, in the same manner as it has been done in [7] for the LEP modes in two identical active circular cavities. Indeed, the field function symmetry or anti-symmetry across the $x$-axis can be imposed by the conditions

$$
U\left(r_{s}, \varphi_{s}\right)= \pm U\left(r_{s},-\varphi_{s}\right),
$$

and the symmetry or anti-symmetry across the $y$-axis that is imposed by the conditions

$$
U\left(r_{s}, \varphi_{s}\right)= \pm U\left(r_{s}, \pi-\varphi_{s}\right) .
$$

The following formulas show the obtained representations of the internal field, where the superscripts $E$ and $O$ indicate the symmetry and anti-symmetry across the $x$-axis and $y$-axis, respectively.

$$
\begin{aligned}
& U^{E E}\left(r_{s}, \varphi_{s}\right)=c_{0} J_{0}\left(k^{\prime} r\right)+2 \sum_{n=0}^{\infty} c_{2 n} J_{2 n}\left(k^{\prime} r\right) \cos (2 n \varphi) \\
& U^{E O}\left(r_{s}, \varphi_{s}\right)=2 i \sum_{n=0}^{\infty} c_{2 n+1} J_{2 n+1}\left(k^{\prime} r\right) \sin ((2 n+1) \varphi) \\
& U^{O E}\left(r_{s}, \varphi_{s}\right)=2 \sum_{n=0}^{\infty} c_{2 n+1} J_{2 n+1}\left(k^{\prime} r\right) \cos ((2 n+1) \varphi) \\
& U^{O O}\left(r_{s}, \varphi_{s}\right)=c_{0} J_{0}\left(k^{\prime} r\right)+2 i \sum_{n=0}^{\infty} c_{2 n} J_{2 n}\left(k^{\prime} r\right) \sin (2 n \varphi)
\end{aligned}
$$

Similar representations are valid for the external field (2) as well, because of the same nature of the dependence of cylindrical functions on their argument's sign.

Simultaneously these symmetries can be represented via conditions on coefficients. They are: all odd coefficients must be zero for the second and third families of symmetry, and similarly all even ones have to vanish for the first and forth families. Additionally for the first and second families all coefficients with opposite-sign indices must differ in sign for the third and forth families.

The series (2) for the external field is not convenient because of the presence of arguments expressed in different origins. To bring formulation to the global-coordinate origin we apply the addition theorem for cylindrical functions,

$$
H_{n}^{(1)}\left(k \rho_{s}\right) e^{i n \varphi_{s}}=\sum_{(l)} H_{n-l}^{(1)}(k s p) J_{l}(k \rho) e^{i n \varphi} .
$$

This leads to the representation that contains infinite sums of the Hankel functions:

$$
U^{\text {out }}(\rho, \varphi)=\sum_{(n)} b_{n} H_{n}^{(1)}(k \rho) e^{i n \varphi}+\sum_{(n)} b_{n} \sum_{s^{\prime} \neq 0} \sum_{(l)} H_{n-l}^{(1)}(k s p) J_{l}(k \rho) e^{i l \varphi} .
$$

Continuity conditions on cylinder's boundary bring us to two coupled infinite matrix equations with the set of unknowns $\left\{c_{m}\right\}_{m=-\infty}^{\infty}$ and $\left\{b_{m}\right\}_{m=-\infty}^{\infty}$,

$$
\begin{aligned}
& c_{m} J_{m}\left(k^{\prime} r\right)=b_{m} H_{m}^{(1)}(k r)+\sum_{(n)} b_{n} J_{m}(k r) D_{m n}, \\
& k c_{m} J_{m}^{\prime}\left(k^{\prime} r\right)=k^{\prime} b_{m} H_{m}^{(1)^{\prime}}(k r)+k^{\prime} \sum_{(n)} b_{n} J_{m}^{\prime}(k r) D_{m n},
\end{aligned}
$$

where so-called lattice sums are 


$$
D_{m n}=\sum_{s \neq 0} H_{n-m}^{(1)}(k s p)= \begin{cases}2 \sum_{s>1} J_{n-m}(2 s k p), & n-m=2 l+1 \\ i 2 \sum_{s>1} Y_{n-m}((2 s+1) k p), & n-m=2 l\end{cases}
$$

Note that if we fix the origin of the global coordinates (i.e., the 0-th cylinder centre) and increase period to infinity $(p \rightarrow \infty)$, then all $D_{m n}=0$, and the coupled matrix equations (8) reduce to the well-known characteristic equation for one dielectric cylinder of the material with the wavenumber $k^{\prime}$ placed in the medium with the wavenumber $k$,

$$
F_{m}(k a, v) \equiv k^{\prime} J_{m}\left(k^{\prime} a\right) H_{m}^{(1)^{\prime}}(k a)-k J_{m}^{\prime}\left(k^{\prime} a\right) H_{m}^{(1)}(k a)=0
$$

Now it is easy to exclude the coefficients $c_{n}$ from the equations (4) and (5) that leads to

$$
0=-F_{m} b_{m}+\left[k J_{m}^{\prime}\left(k^{\prime} a\right) J_{m}(k a)-k^{\prime} J_{m}\left(k^{\prime} a\right) J_{m}^{\prime}(k a)\right] \sum_{(n)} b_{n} D_{m n}
$$

On introducing new unknowns, $\left\{x_{m}\right\}_{m=-\infty}^{\infty}$, according to the formula

$$
b_{m}=x_{m} J_{m}(k a) / F_{m} J_{m}\left(k^{\prime} a\right),
$$

we obtain the final infinite-matrix equation:

$$
[I+A(\kappa, \gamma ; \xi, \alpha)] X=0, \quad A=\left\{A_{m n}\right\}_{m . n=-\infty}^{+\infty}
$$

where $\kappa=k a, \quad \xi=p / a$, and

$$
\begin{gathered}
A_{m n}=K_{m n}(\kappa, \gamma ; \alpha) D_{m n}(\kappa \xi), K_{m n}(\kappa, \gamma ; \alpha)=\frac{V_{m}(\kappa, v) J_{n}(\kappa)}{F_{n}(\kappa, v) J_{n}(\kappa v)} \\
V_{m}(\kappa, v)=k J_{m}^{\prime}(\kappa v) J_{m}(\kappa)-k v J_{m}(\kappa v) J_{m}^{\prime}(\kappa)
\end{gathered}
$$

This is the Fredholm second-kind matrix equation due to the rapid enough decrease of the matrix elements $A_{m n}$ if $n, m \rightarrow \infty$. Therefore its determinant exists as a function of parameters, and the characteristic equation for the eigenvalues is

$$
\operatorname{Det}[I+A(\kappa, \gamma ; \xi, \alpha)]=0
$$

The discussed above separation of the lasing modes to four symmetry families leads to the independent determinantal equations generated by the similar second-kind matrices having the following elements:

$$
\begin{aligned}
& \text { for the EE and EO modes: } \left.A_{m n}^{E J}= \pm \mu_{n} K_{m n}(\kappa, \gamma) \sum_{(s)}\left[H_{m+n}^{(1)}(s \kappa \xi)+(-1)^{p} H_{m-n}^{(1)}(s \kappa \xi)\right], \quad J=E, O, \quad m, n=0,1, \ldots,(17)\right) \\
& \text { for the OE and OO modes: } A_{m n}^{O J}= \pm K_{m n}(\kappa, \gamma) \sum_{(s)}\left[H_{m+n}^{(1)}(s \kappa \xi)-(-1)^{p} H_{m-n}^{(1)}(s \kappa \xi)\right], \quad J=E, O, \quad m, n=1,2, \ldots,
\end{aligned}
$$

where $\mu_{0}=1 / 2, \mu_{p>0}=1$.

Note that in each family, the eigenvalues are located in the union of circles on the plane $(\kappa, \gamma)$ defined by the expressions

$$
\left|1+A_{m m}^{I J}(\kappa, \gamma ; \xi, \alpha)\right|<\sum_{n \neq m}\left|A_{m n}^{I J}(\kappa, \gamma ; \xi, \alpha)\right|^{2}, m=(0) 1,2, \ldots, I=E, O, \quad J=O, E
$$

This is the consequence of the Gershgorin theorem in the matrix eigenvalue theory [8].

Representation of the external field (2) in the form of series using the Hankel functions is slowly convergent; the way out is to rewrite the field in terms of the Floquet harmonics - see [9-13]. This is achieved by substituting the Fourier transforms for the Hankel functions,

$$
H_{n}^{(1)}\left(k r_{s}\right) e^{i n \varphi_{s}}=\frac{1}{\pi i^{n}} \int_{-\infty}^{\infty} e^{\left(i k(x-s p) t+i k y \sqrt{1-t^{2}}\right)} \frac{e^{i n \sin ^{-1} t}}{\sqrt{1-t^{2}}} d t
$$

into expression (2). As a result, we obtain the Floquet series in terms of generalized plane waves,

$$
U^{\text {out }}(r, \varphi)=\sum_{(s)} u_{s}^{ \pm} e^{i \frac{2 s \pi}{p} x} e^{i g_{s}|y|},|y|>a
$$

where $g_{s}=\sqrt{k^{2}+(2 s \pi / p)^{2}}$ and the amplitudes of Floquet harmonics are 


$$
u_{s}^{ \pm}=\frac{2}{g_{s} p} \sum_{(n)}(\mp i)^{n} b_{n} 2 e^{i n \sin ^{-1} \frac{2 s \pi}{k p}}
$$

The modal symmetry or anti-symmetry across the $x$-axis effects enter into (22) via the corresponding parity properties of coefficients $b_{n}$.

\section{CONCLUSIONS}

In this paper the mathematical approach to the LEP for an infinite periodic chain of active circular cylinders has been considered. Determinantal equations for the lasing modes of different symmetry classes have been derived based on the Fredholm second kind infinite-matrix equations. The field function representations in terms of the Floquet harmonics have been obtained for the space domains above and below the grid of cylinders. These results form the mathematical basis for building a numerical algorithm for the search of the LEP eigenvalues (frequencies and thresholds) of periodic chain of active circular cavities or quantum wires.

\section{ACKNOWLEDGEMENTS}

This work was supported in part by the National Academy of Sciences of Ukraine (NASU) via project \#07/36-H, the Royal Society, UK via project IJP-2007/R1-FS, and the Turkish Science and Technology Research Council via exchange programme. V.O.B. was also supported by the IEEE AP Society with a graduate student fellowship award.

\section{REFERENCES}

[1] S. F. Yu, Analysis and Design of Vertical Cavity Surface Emitting Lasers, Wiley, 2003.

[2] J.V. Campenhout, P. Bienstman, R. Baets, "Band-edge lasing in gold-clad photonic-crystal membranes", IEEE J. Selected Areas in Communications, vol. 23, no 7, pp. 1418-1423, 2005.

[3] A.I. Nosich, E.I. Smotrova, S.V. Boriskina, T.M. Benson, P. Sewell, "Trends in microdisk laser research and linear optical modelling”, Optical and Quantum Electronics, vol. 39, no 15, pp. 1253-1272, 2007.

[4] E.I. Smotrova, A.I. Nosich, "Mathematical analysis of the lasing eigenvalue problem for the WG modes in a 2-D circular microcavity", Optical and Quantum Electronics, vol. 36, no 1-3, pp. 213-221, 2004.

[5] E.I. Smotrova, A.I. Nosich, T.M. Benson, P. Sewell, "Cold-cavity thresholds of microdisks with uniform and non-uniform gain: quasi-3D modelling with accurate 2D analysis", IEEE J. Selected Topics Quantum Electronics, vol. 11, no 5, pp. 1135-1142, 2005.

[6] V.O. Byelobrov, A.I. Nosich, "Mathematical analysis of the lasing eigenvalue problem for the optical modes in a layered dielectric microcavity with a quantum well and distributed Bragg reflectors", Optical and Quantum Electronics, vol. 39, no 10-11, pp. 927-937, 2007.

[7] E.I. Smotrova, et al., Optical coupling of the WG modes of two identical microdisks and its effect on photonic molecule lasing, IEEE J. Selected Topics Quantum Electronics, vol. 12, no 1, pp. 78-85, 2006.

[8] J.R. Westlake, A Handbook of Numerical Matrix Inversion and Solution of Linear Equations, NY, Wiley, 1968.

[9] V. Twersky, On scattering of waves by the infinite grating of circular cylinders, IRE Trans. Antennas Propagation, no 10, pp. 737-765, 1962.

[10] N. Nicorovici, R. McPhedran, "Efficient calculation of the Green's function for electromagnetic scattering by gratings", Phys. Rev. E, vol. 50, no 4, pp. 3143-3160, 1994.

[11] C.M. Linton, "The Green's function for the two-dimensional Helmholtz equation in periodic domains", J. Engineering Mathematics, vol. 33, pp. 377-402, 1998.

[12] Ö. Kavaklioglu, "On diffraction of waves by the infinite grating of circular dielectric cylinders at oblique incidence: Floquet representation”, J. Modern Optics, vol. 48, no 1, pp. 125-142, 2001.

[13] K. Yasumoto, H. Toyama, T. Kushta, "Accurate analysis of 2-D electromagnetic scattering from multilayered periodic arrays of circular cylinders using lattice sums technique", IEEE Trans. Antennas Propagation, vol. 52, no 10, pp. 2603-2611, 2004. 